1. Rogers, D. P. \& Bankaitis, V. A. Phospholipid transfer proteins and physiological functions. Int. Rev. Cytol. 197, 35-81 (2000).

2. Wirtz, K. W. A. Phospholipid transfer proteins revisited. Biochem. J. 324, 353-360 (1997).

3. Zhou, D. et al. Editing of CD1d-bound lipid antigens by endosomal lipid transfer proteins. Science 303, 523-527 (2004).

4. Hakomori, S. The glycosynapse. Proc. Natl Acad. Sci. USA 99, 225-232 (2002).

5. Dwek, R. A., Butters, T. D., Platt, F. M. \& Zitzmann, N. Targeting glycosylation as a therapeutic approach. Nature Rev. Drug Discov. 1, 65-75 (2002).

6. Metz, R. J. \& Radin, N. S. Purification and properties of a cerebroside transfer protein. J. Biol. Chem. 257, 12901-12907 (1982)

. Abe, A. \& Sasaki, T. Purification and some properties of the glycolipid transfer protein from pig brain. J. Biol. Chem. 260, 11231-11239 (1985).

8. Brown, R. E., Jarvis, K. L. \& Hyland, K. J. Purification and characterization of glycolipid transfer protein from bovine brain. Biochim. Biophys. Acta 1044, 77-83 (1990).

9. Yamada, K., Abe, A. \& Sasaki, T. Specificity of the glycolipid transfer protein from pig brain. J. Biol. Chem. 260, 4615-4621 (1985).

10. Lin, X., Mattjus, P., Pike, H. M., Windebank, A. J. \& Brown, R. E. Cloning and expression of glycolipid transfer protein from bovine and porcine brain. J. Biol. Chem. 275, 5104-5110 (2000).

11. Brodersen, P. et al. Knockout of Arabidopsis accelerated-cell-death11 encoding a sphingosine transfer protein causes activation of programmed cell death and defense. Genes Dev. 16, 490-502 (2002).

12. Mattjus, P., Turcq, B., Pike, H. M., Molotkovsky, J. G. \& Brown, R. E. Glycolipid intermembrane transfer is accelerated by HET-C2, a filamentous fungus gene product involved in the cell-cell incompatibility response. Biochemistry 42, 535-542 (2003).

13. Tsujishita, Y. \& Hurley, J. H. Structure and lipid transport mechanism of a StAR-related domain. Nature Struct. Biol. 7, 408-414 (2000).

14. Roderick, S. L. et al. Structure of human phosphatidylcholine transfer protein in complex with its ligand. Nature Struct. Biol. 9, 507-511 (2002).

15. Min, K. C., Kovall, R. A. \& Hendrickson, W. A. Crystal structure of $\alpha$-tocopherol transfer protein bound to it ligand: implications for ataxia with vitamin E deficiency. Proc. Natl Acad. Sci. USA 100, 14713-14718 (2003).

16. Gadola, S. D. et al. Structure of human CDlb with bound ligands at $2.3 \AA$, a maze of alkyl chains. Nature Immunol. 3, 721-726 (2002).

17. Zajonc, D. M., Elsliger, M. A., Teyton, L. \& Wilson, I. A. Crystal structure of CD1a in complex with a sulfatide self antigen at a resolution of 2.15 A. Nature Immunol. 4, 808-815 (2003).

18. Mahfoud, R. et al. Identification of a common sphingolipid-binding domain in Alzheimer, prion, and HIV-1 proteins. J. Biol. Chem. 277, 11292-11296 (2002).

19. Schubert Wright, C., Zhao, Q. \& Rastinejad, F. Structural analysis of lipid complexes of GM2-activator protein. J. Mol. Biol. 331, 951-964 (2003).

20. Weis, W. I. \& Drickamer, K. Structural basis of lectin-carbohydrate interactions. Annu. Rev. Biochem. 65, 441-473 (1996).

21. Feinberg, H., Mitchell, D. A., Drickamer, K. \& Weis, W. I. Structural basis for selective recognition of oligosaccharides by DC-SIGN and DC-SIGNR. Science 294, 2163-2166 (2001).

22. Wimley, W. W. \& White, S. H. Experimentally determined hydrophobicity scale for proteins at membrane interfaces. Nature Struct. Biol. 3, 842-848 (1996).

23. Killian, J. A. \& von Heijne, G. How proteins adapt to a membrane-water interface. Trends Biochem. Sci. 25, 429-434 (2000)

24. Doublié, S. Preparation of selenomethionyl proteins for phase determination. Methods Enzymol. 276, 523-530 (1997).

25. Li, X.-M., Momsen, M. M., Brockman, H. L. \& Brown, R. E. Lactosylceramide: effect of acyl chain structure on phase behavior and molecular packing. Biophys. J. 83, 1535-1546 (2002).

26. Hendrickson, W. A. Determination of macromolecular structures from anomalous diffraction of synchrotron radiation. Science 254, 51-58 (1991).

27. Terwilliger, T. C. \& Berendzen, J. Automated MAD and MIR structure solution. Acta Crystallogr. D 55, 849-861 (1999).

28. Lamzin, V. S. \& Wilson, K. S. Automated refinement of protein models. Acta Crystallogr. D 49, 129-149 (1993).

29. Murshudov, G. N., Vagin, A. A. \& Dodson, E. J. Refinement of macromolecular structures by the maximum-likelihood method. Acta Crystallogr. D 53, 240-255 (1997).

30. Navaza, J. AMoRe: an automated package for molecular replacement. Acta Crystallogr. A 50, 157-163 (1997).

Supplementary Information accompanies the paper on www.nature.com/nature.

Acknowledgements We thank the personnel at SBC beamline 19BM of the Advanced Photon Source beamline staff for assistance with data collection from multiwavelength anomalous dispersion; A. Serganov for technical support; X. Lin, T. Chung and H. Pike for their contributions to the cloning and expression of the recombinant human GLTP; X.-M. Li for synthesizing and purifying N-18:1 lactosylceramide; A. J. Windebank for help with DNA sequencing at the Mayo Molecular Biology Core Facility; T. Burghardt for help with recording near-ultraviolet CD spectra; and S. Venyaminov in the Franklyn Prendergast laboratory for recording the far-ultraviolet $\mathrm{CD}$ spectra. This research was supported by NIH and the Hormel Foundation. Use of the ANL SBC beamlines at the APS was supported by the US Department of Energy, Office of Energy Research.

Competing interests statement The authors declare that they have no competing financial interests.

Correspondence and requests for materials should be addressed to R.E.B. (reb@umn.edu) or D.P. (pateld@mskcc.org).Coordinates have been deposited in the Protein Data Bank under accession codes 1SWX for apo-GLTP and 1SX6 for lactosylceramide-bound GLTP.

\section{Structural rearrangements in the membrane penetration protein of a non-enveloped virus}

\author{
Philip R. Dormitzer ${ }^{1}$, Emma B. Nason ${ }^{3}$, B. V. Venkataram Prasad \\ \& Stephen C. Harrison ${ }^{1,2}$
}

${ }^{1}$ Department of Pediatrics, Harvard Medical School, and the Laboratory of Molecular Medicine, Children's Hospital, and

${ }^{2}$ Howard Hughes Medical Institute, 320 Longwood Avenue, Boston, Massachusetts 02115, USA

${ }^{3}$ Verna and Marrs McLean Department of Biochemistry and Molecular Biology, Baylor College of Medicine, One Baylor Plaza, Houston, Texas 77030, USA

Non-enveloped virus particles (those that lack a lipid-bilayer membrane) must breach the membrane of a target host cell to gain access to its cytoplasm. So far, the molecular mechanism of this membrane penetration step has resisted structural analysis. The spike protein VP4 is a principal component in the entry apparatus of rotavirus, a non-enveloped virus that causes gastroenteritis and kills 440,000 children each year ${ }^{1}$. Trypsin cleavage of VP4 primes the virus for entry by triggering a rearrangement that rigidifies the VP4 spikes ${ }^{2}$. We have determined the crystal structure, at $3.2 \AA$ resolution, of the main part of VP4 that projects from the virion. The crystal structure reveals a coiledcoil stabilized trimer. Comparison of this structure with the two-fold clustered VP4 spikes in a $\sim 12 \AA$ resolution image reconstruction from electron cryomicroscopy of trypsin-primed virions shows that VP4 also undergoes a second rearrangement, in which the oligomer reorganizes and each subunit folds back on itself, translocating a potential membrane-interaction peptide from one end of the spike to the other. This rearrangement resembles the conformational transitions of membrane fusion proteins of enveloped viruses ${ }^{3-6}$.

VP4 spikes on virions have 'head', 'body', 'stalk' and 'foot' regions (Fig. 1a), formed by the two VP4 trypsin cleavage fragments VP8* and $\mathrm{VP}^{\star}{ }^{\star}$ (Fig. 1b). The VP8* fragment contains a globular domain, the 'VP8* core', which forms the head'. In some virus strains the $\mathrm{VP}^{*}$ core is a haemagglutination domain, which mediates initial cell attachment by binding sialic acid. Portions of both VP8* and $\mathrm{VP}^{\star}$ make up the body. VP5* residues in the body are implicated in membrane penetration and integrin binding ${ }^{8-10}$. The carboxyterminal part of $\mathrm{VP}^{\star}{ }^{\star}$ forms the foot, which is buried beneath the VP7 layer, anchoring the spike. Neutralizing antibodies against rotavirus bind $\mathrm{VP}^{\star}, \mathrm{VP}^{\star}$ and VP7 and block cell entry ${ }^{11}$. When present in the gut lumen, they protect against rotavirus gastroenteritis ${ }^{12}$. Thus, knowledge of the structural basis for cell entry can inform vaccine development.

The $\mathrm{VP}^{*}$ fragment used for structure determination (VP5CT; Fig. 1b) is produced by serial chymotrypsin and trypsin cleavage of a VP4 precursor ${ }^{13}$. Expressed directly, the fragment is insoluble and probably misfolded. VP5CT has the authentic VP5* amino terminus at A248. VP4 cleavage to produce this $\mathrm{N}$ terminus is required to prime particles for cell entry and membrane interaction ${ }^{14,15}$. The crystal structure of VP5CT contains rhesus rotavirus (RRV) VP4 residues N252-L523. Perfect hemihedral twinning prevented use of diffraction data beyond $3.2 \AA$ resolution in the structure determination (see Methods and Supplementary Methods).

VP5CT is a well-ordered homotrimer that resembles a folded umbrella (Fig. 2a). The 'post' of the umbrella is a C-terminal, $\alpha$-helical, triple coiled-coil. Each of the three panels comprising the 'shade' of the umbrella is an N-terminal globular domain (Fig. 2b). Each globular domain packs in a groove between the $\alpha$-helices of the other two subunits on the post's negatively charged outer surface 
(Fig. 2c). Just above the post, strands $\mathrm{K}, \mathrm{H}$, and $\mathrm{G}$ from each globular domain join in a continuously hydrogen-bonded $\beta$-annulus around the three-fold axis (Fig. 2a). At the top of the structure, propeller-like packing of tryptophan side chains (W262, strand B) creates an additional trimer contact. Between the $\beta$-annulus and the W262 propeller, a cavity (volume $390 \AA^{3}$ ) centred on the three-fold axis leaves room for potential rearrangements. In full-length VP5*, the foot region (Fig. 1a), similar in mass to VP5CT, is attached to the coiled-coil.

The core of each globular domain is an eight-stranded antiparallel $\beta$-sandwich (Fig. 2b, light and dark blue). Two features of potential functional importance project from its top edge: the $\mathrm{GH}$ and $\mathrm{CD} \beta$-hairpins. By joining with strands $\mathrm{K}$ in the $\beta$-annulus, the GH hairpin clamps each globular domain in the 'folded umbrella' position. The flexible tip of the $\mathrm{CD} \beta$-hairpin bears a sequence motif (DGE) implicated in rotavirus binding to $\alpha 2 \beta 1$ integrins (Fig. 2b, e $)^{10}$. The accessibility of the motif, which protrudes into solvent, supports the proposed receptor-binding function.

The tips of loops projecting from the bottom edge of the $\beta$-sandwich impart a hydrophobic apex to the globular domain (Fig. 2d), which may function in membrane penetration. Near the $\beta$-sandwich, portions of these loops form $\beta$-sheets $F^{\prime} G\left(H / H^{\prime}\right)$ and $\mathrm{B}^{\prime} \mathrm{C}^{\prime} \mathrm{E}^{\prime} \mathrm{D}^{\prime}$ (purple and pink respectively in Fig. 2b). The unusual interposition of $\beta$-strand I (green) between these sheets creates a deep 'hydrophobic bowl'. The distal part of the $F^{\prime} G$ loop and the alphavirus 'fusion loop' have similar sequences ${ }^{8}$. Both loops lie at the hydrophobic tips of globular domains implicated in membrane disruption, but the domains and the loops themselves are otherwise structurally dissimilar (Supplementary Fig. 1 and Tables 2, 3) ${ }^{6}$. Thus, the sequence similarity probably reflects selection for hydrophobicity and flexibility in both loops, but not common ancestry. Each strand of the $\mathrm{F}^{\prime} \mathrm{G}$ loop has an in-register glycine-glycine pair (382-383 and 399-400, located under labels $\mathrm{F}^{\prime}$ and $\mathrm{G}$ in Fig. 2b).
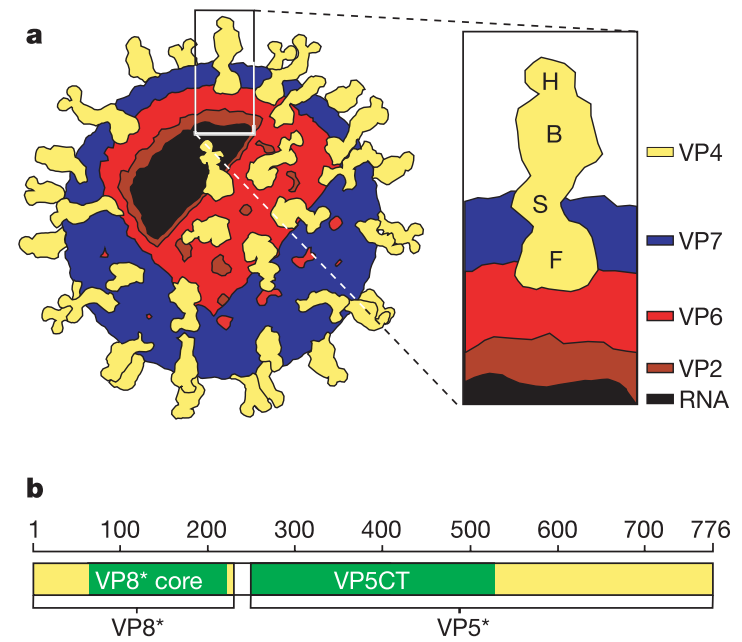

Figure 1 Rotavirus virion structure, VP4 domains and fragments. a, The icosahedral virion has three layers: an inner VP2 layer, which contains the genome, polymerase and capping enzyme; a middle VP6 layer; and an outer VP7 layer. To initiate infection, a 710 A diameter subviral particle, consisting of the inner two layers and their contents, is delivered into the cytoplasm. Head (H), body (B), stalk (S) and foot (F) regions of the VP4 spikes are indicated. The approximate two-fold axis of the head and body does not correspond to any icosahedral symmetry axis. The protruding portion of the spike is within the icosahedral asymmetric unit. The drawing is based on an electron cryomicroscopy image reconstruction ${ }^{30}$. b. Diagram illustrating the VP8* (residues M1-R231) and VP5* (A248-L776) fragments, produced by priming virions with trypsin ${ }^{14}$; the VP5CT fragment (A248-L525 to F528), produced by serially digesting soluble VP4 with chymotrypsin and trypsin ${ }^{13}$; and the VP8* core domain (L65-L224)
The four conserved glycines might provide a hinge, opening up the hydrophobic bowl beneath and allowing relatively extensive and flexible membrane interactions during cell entry.

How does the VP5CT structure relate to that of the VP4 spike? The region of VP5CT from Y267 to L470 neatly fits the molecular envelope of the spike body in a new, $\sim 12 \AA$ resolution electron cryomicroscopy image reconstruction of trypsin-primed virions (Fig. 3b, e). As Y267 and L470 hydrogen bond to each other on adjacent $\beta$-strands $B$ and $J$, this region constitutes a self-contained globular domain called the VP5 ${ }^{\star}$ antigen domain (solid colours in Fig. 3c, f). VP5CT residues outside the antigen domain must fold differently in the spike than in the trimeric crystal structure (white outlines in Fig. 3c, f).

Together, the $\mathrm{VP}^{\star}$ antigen domain and the $\mathrm{VP} 8^{\star}$ core contain all known neutralizing epitopes on VP4 (Fig. 3a, d), making them logical minimal antigens for recombinant rotavirus vaccines. The much greater conservation in $\mathrm{VP}^{\star}{ }^{\star}$ suggests stronger functional constraints and correlates with heterotypic neutralization by some $\mathrm{VP} 5^{\star}$-specific antibodies. The $\mathrm{VP} 5^{\star}$ antigen domain is thus a particularly promising potential immunogen. On the basis of the VP5CT structure, we have grouped VP5 ${ }^{\star}$ neutralization escape mutations into five epitopes (Supplementary Table 1). The epitopes are solvent-exposed on both the VP5CT trimer (Fig. 2c, d) and the spike body (Fig. 3a, d). The surfaces of the trimer that lack neutralization epitopes are inaccessible on the spike, explaining their antigenic 'silence' on primed virions.

Most VP5 ${ }^{\star}$-specific neutralizing monoclonal antibodies map to epitope 5-1, the exposed surface of the 'shoulder' at the virion-distal end of the spike body, formed by the hydrophobic apex of the antigen domain (Fig. 3). The head covers much of the apex, but part of the $\mathrm{F}^{\prime} \mathrm{G}$ loop is exposed. Monoclonal antibody $2 \mathrm{G} 4$ selects a neutralization escape mutation in this loop at residue Q393 (ref. 8). Electron cryomicroscopy of antibody-binding fragment (FAb)decorated virions shows that $2 \mathrm{G} 4$ indeed binds the shoulder ${ }^{16}$, confirming our fit of the antigen domain to the spike body. Antibody 2G4 blocks a post-attachment entry event ${ }^{11}$, which is possibly domain rotation or membrane insertion (see below). Epitopes 5-1, 5-2, 5-3 and 5-4 are linked by a network of antibody competition and escape mutant cross-resistance (references in Supplementary Table 1). Epitope 5-5 is outside of this network and is mimicked by a short synthetic peptide ${ }^{17}$. It maps to the flexible CD hairpin, adjacent to the integrin-binding motif (Fig. 2b, c, e), suggesting that epitope 5-5-specific antibodies may block integrin interactions.

Image reconstructions from electron cryomicroscopy of rotavirus particles have provided good evidence for two conformational states of VP4: an uncleaved, flexible state, which does not produce visible density in averaged image reconstructions ${ }^{2}$ (Fig. 4a), and a trypsin-cleaved, rigid state with approximate two-fold symmetry of the parts that project beyond the VP7 shell (Figs 3b, e and 4b) ${ }^{18,19}$. Contrary to the two-fold character of the projecting part of the spikes on trypsin-primed virions, the VP5CT crystal structure reveals an unambiguous trimer (Fig. 2a, c, d). The three-fold contacts bury $3,956 \AA^{2}(25.8 \%)$ of the surface of each subunit and render the trimer resistant to dissociation by SDS $^{13}$. Such extensive and stable contacts provide structural evidence that this region of VP4 evolved to trimerize. VP5CT thus represents a third conformational state for the projecting part of VP4 (Fig. 4c).

How can a two-fold to three-fold reorganization of VP4 occur? For the transition to take place on the virion, rather than after dissociation, each spike location must actually contain three VP4 subunits at all stages. Indeed, recently obtained image reconstructions of trypsinized rotavirus particles treated briefly at $\mathrm{pH} 11$ reveal foreshortened spikes with clear three-fold character: trefoil-like features projecting slightly above the VP7 layer (B.V.V.P., manuscript in preparation). Unfolding at elevated $\mathrm{pH}$ appears to cause each VP4 subunit to condense so that better-ordered segments near 
the foot produce strong density in the image. We conclude that twofold clustering after trypsin cleavage leaves the third subunit still flexible, as in the uncleaved state (Fig. 4b).

Several observations support this view of VP4 organization. The spike projects from an asymmetric, angled stalk, so that its approximate two-fold axis is displaced from the centre of the foot ${ }^{18,19}$. Thus, the symmetry of the foot and the projecting spike need not match. Indeed, image reconstructions of VP4 obtained by subtracting images of spikeless particles from images of particles with spikes reveal three-fold symmetry in the foot ${ }^{19}$. The symmetry mismatch between foot and projection was previously reconciled by proposing that two VP4 subunits each contribute three similarly shaped domains to the foot. The new data suggest, instead, that the three-fold symmetry of the foot reflects the true subunit stoichiometry. Our previous biochemical data on VP5CT oligomers in solution were consistent either with trimers or with tight dimers $\mathbf{a}$

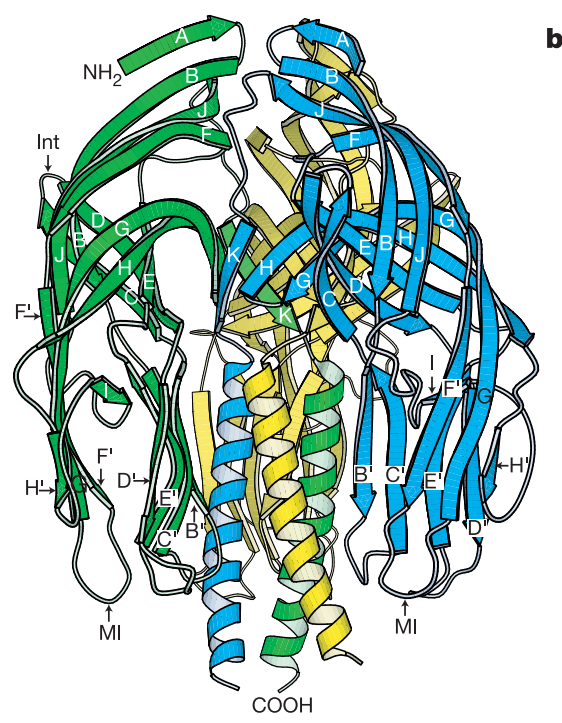

c

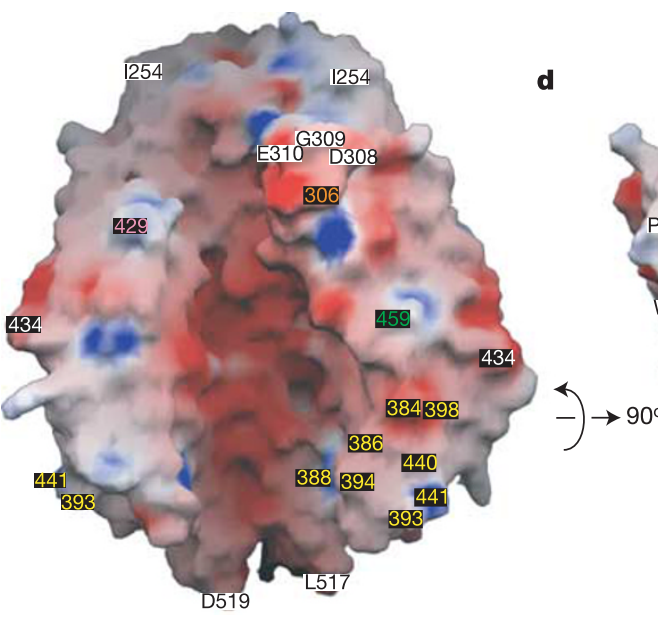

e

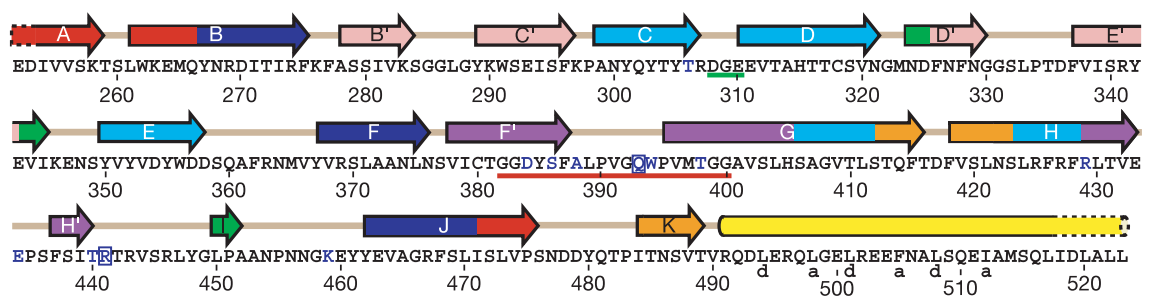

Figure 2 VP5CT structure. a, Ribbon diagram of VP5CT. The trimer stands $84 \AA$ high with a $37 \AA$ maximum radius. The green and blue subunits depict residues I254-D519; the yellow subunit depicts residues E252-L517. Int, integrin-binding motif; MI, potential membrane interaction $\left(F^{\prime} G\right)$ loop. $\mathbf{b}$, Ribbon diagram of a single VP5CT subunit (I254-L523). The colours of the secondary structure elements match those in e. c. The VP5CT surface oriented as in a and coloured by electrostatic potential: blue, positive; red, negative. Surfaces formed by residues selected in neutralizing antibody escape mutants (Supplementary Table 1) are labelled with residue numbers coloured by epitope in black text boxes: 5-1, yellow; 5-2, white; 5-3, green; 5-4, pink; 5-5, orange. I254, L517 and D519 mark N- and C termini of the depicted subunits. D308, G309 and E310 form the b

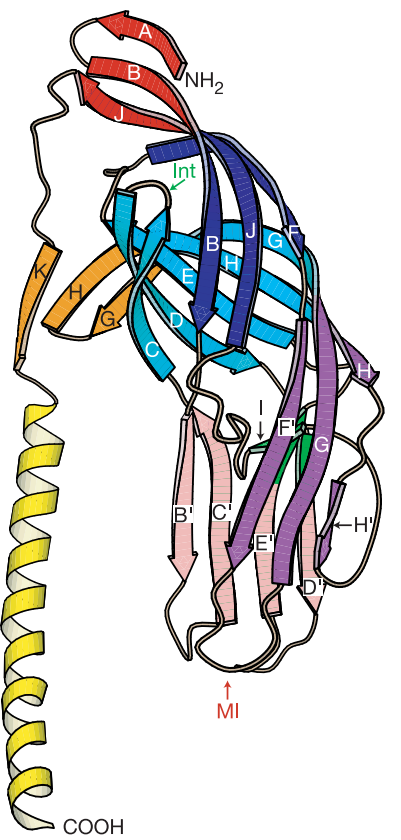

integrin-binding motif at the tip of the claw-like CD hairpin. d, The VP5CT surface, coloured as in c and viewed along the three-fold symmetry axis from the bottom. Surfaces formed by hydrophobic residues at the apex of the globular domain are labelled on the upper-left subunit. e, Secondary structure assigned to the RRV primary amino acid sequence. $\beta$-strands are arrows; the $\alpha$-helix is a tube. Variable secondary structure at the $\mathrm{N}$ - and $\mathrm{C}$ termini is indicated by dashed outlines. Blue letters indicate neutralization escape mutations; escape mutations in strain RRV are boxed ${ }^{8}$. The green underline identifies the integrin-binding motif ${ }^{10}$. The red underline identifies the potential membrane interaction loop ${ }^{8}$. Residues that make hydrophobic contacts around the three-fold axis of the coiled-coil are indicated by 'a' or 'd', corresponding to heptad repeat positions. 

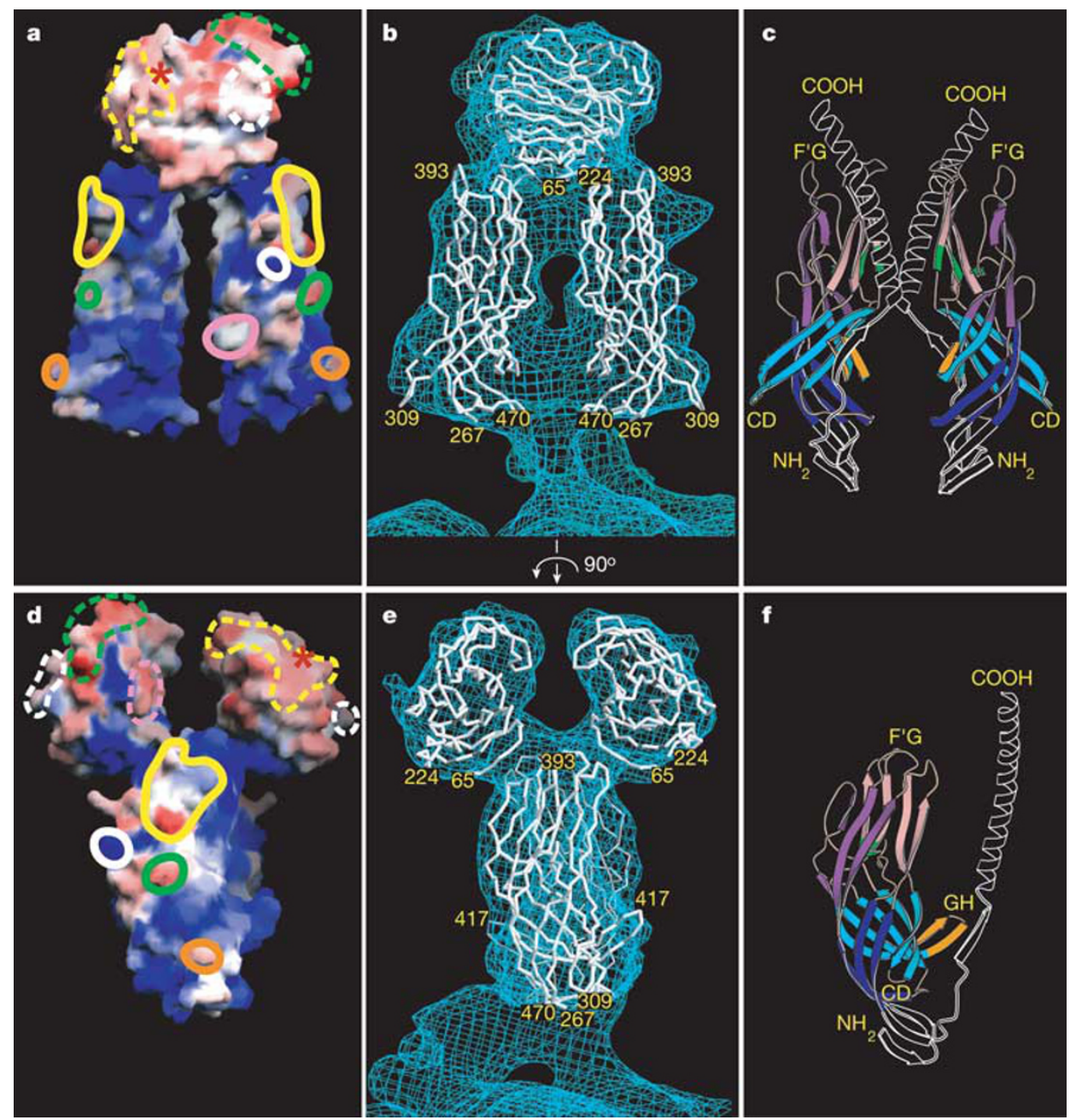

Figure 3 The VP5* antigen domain and the VP8* core fit to an electron cryomicroscopy image reconstruction. Each model in $\mathbf{d}-\mathbf{f}$ is rotated $90^{\circ}$ about a vertical axis relative to the corresponding model in a-c. a, d, Surface models oriented as in the spike. The surfaces are coloured by conservation in $20 \mathrm{P}$ genotypes (sequences referenced in Supplementary Table 2): blue, conserved; red, variable. Neutralization epitopes are outlined: 5-1, solid yellow; 5-2, solid white; 5-3, solid green; 5-4, solid pink; 5-5, solid orange; 8-1, dashed yellow; 8-2, dashed white; 8-3, dashed green; 8-4, dashed pink. VP8* epitopes and the sialoside-binding site (red asterisk) have been described previously ${ }^{7} . \mathbf{b}, \mathbf{e}, \mathrm{C} \alpha$ traces of the $\mathrm{VP}^{*}$ core and VP5* antigen domain fit to an image reconstruction of trypsinized virions at $\sim 12 \AA$ resolution, contoured at $0.45 \sigma$. Labelled residues: 65 and 224, VP8* core termini; 267 and $470, \mathrm{VP}^{*}$ antigen domain termini; 309, glycine of the DGE integrin-binding motif (CD loop); 393, immunodominant site in the potential membrane interaction ( $F^{\prime} G$ ) loop; 417, tip of the GH hairpin. c, f, Ribbon diagrams of the VP5CT subunit, with the antigen domain oriented to fit the spike body. The antigen domain is coloured to match Fig. 2b. Residues outside the antigen domain, which must fold differently in the spike, are drawn in white outline. that associate weakly into tetramers (dimers of dimers) ${ }^{13}$. At the time, the latter interpretation appeared more consistent with electron microscopy, but the crystal structure and new image reconstructions resolve the ambiguity in favour of trimers.

If just two of three VP4 subunits at each spike location cluster after trypsin cleavage, interactions with VP7 and/or VP6 must select the two that form the visible spike. The VP8* and VP5 $5^{\star}$ fragments of the third, flexible molecule need not remain associated, and this subunit could even perform a different function in cell entry. Interactions with VP7 and VP6 may also arrest the progression of VP4 rearrangements at the primed stage.

In the dimeric spike, a loop $\left(\mathrm{F}^{\prime} \mathrm{G}\right)$ with the properties of a membrane-interacting element points away from the foot, and the $\mathrm{GH}$ hairpin points approximately $90^{\circ}$ away from the dyad axis of the body (Fig. 3b, c, e, f). In the VP5CT trimer, the $F^{\prime} G$ loop points towards the $\mathrm{C}$ terminus of the coiled-coil, to which the stalk and foot attach, and the GH hairpin joins the $\beta$-annulus around the three-fold axis (Fig. 2a). Therefore, in the two-fold to three-fold reorganization, the $\mathrm{VP}^{\star}{ }^{\star}$ antigen domain must rotate approximately $180^{\circ}$ about an axis roughly perpendicular to the spike's long axis (Fig. 4b, c). This rotation translocates the hydrophobic tip of the $F^{\prime} G$ loop by at least $55 \AA$ from the shoulder of the spike towards the foot.

The two subunits of the spike body make proximal and distal dyad contacts, leaving a gap in the centre (Fig. 3b, e). The VP4 residues that participate in these contacts are outside the relatively rigid $\mathrm{VP}^{\star}{ }^{\star}$ core and $\mathrm{VP}^{\star}$ antigen domain and probably control their large-scale displacements. Both termini of the VP8 $8^{\star}$ core are in the 'neck' between head and body, near the distal dyad contact. Both termini of the $\mathrm{VP}^{\star}{ }^{\star}$ antigen domain are at the bottom of the body, adjacent to the proximal dyad contact. The antigen domain completely fills the electron microscopy envelope between the dyad contacts, leaving no unfilled density to suggest an unmodelled connecting strand. Therefore, $\mathrm{VP}^{\star}{ }^{\star}$ residues outside the core (M1-V64) must form the distal dyad contact and tether head to body. During rotation of the $\mathrm{VP}^{\star}$ antigen domain, disruption of the distal dyad contact would release VP8 ${ }^{\star}$ (Fig. 4c). Similarly, VP5 ${ }^{\star}$ residues outside the antigen domain (A248-Q266 and I471-L523 and perhaps additional $\mathrm{C}$-terminal residues that connect to the stalk) must form the proximal dyad contact. In the trimeric state, 
a

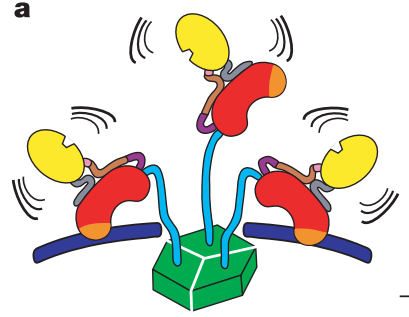

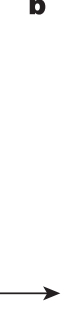

部

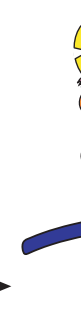

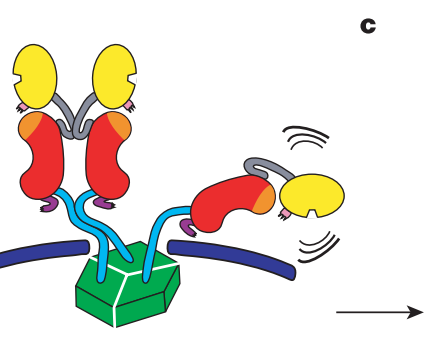

c

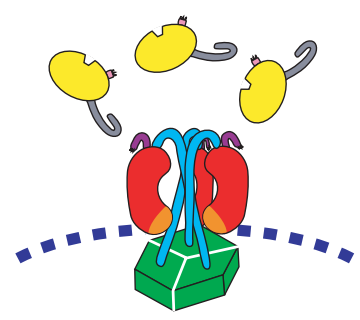

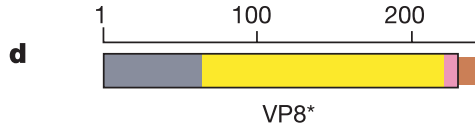

Figure 4 Model for VP4 rearrangements during priming and entry. The colours in the cartoon (a-c) match those in the linear diagram (d). The VP7 shell is blue. $\mathbf{a}$, Uncleaved state. The wavy lines indicate flexibility. $\mathbf{b}$, Trypsin-primed state. The activation region (brown in a) between VP8* and VP5* has been removed by digestion. Two clustered molecules form the visible spike. The head is the yellow oval (the VP8* core), notched at the sialoside-binding cleft. The body includes the red 'kidney' (the VP5* antigen domain), the orange cap (the potential membrane interaction loop), the purple appendage, and part of the grey and cyan tubes. The stalk is the lower part of the cyan tubes. The foot is the green hexagonal prism. c, Folded-back state. This state corresponds to the VP5CT crystal structure and may form during membrane penetration. The dashed blue line indicates potential loss of VP7. d, Linear diagram of structural elements. The VP8* and VP5* fragments are in black boxes. Grey, residues M1-V64; yellow, L65-L224; pink, P225-R231; brown, N232-R247; purple, A248-Q266; red, Y267-T381 and A401-L470; orange, G382-G400; cyan, I471-unknown; green, unknown-L776. these residues make up the elements of the three-fold contacts: $\beta$-sheet ABJ, $\beta$-strand $K$ and the $\alpha$-helix (Figs $2 \mathrm{a}$ and $3 \mathrm{c}, \mathrm{f}$ ). The refolding of residues in the proximal dyad contact to form the coiled-coil may drive the two-fold to three-fold reorganization.

The three distinct conformations of VP4 correspond to steps in rotavirus entry (Fig. 4a-d). When uncleaved virions are released from infected cells, three VP4 molecules with flexible stalks occupy each of 60 symmetry-equivalent positions (Fig. 4a). In the gut lumen, trypsin cleavage between $\mathrm{VP}^{\star}$ and $\mathrm{VP} 5^{\star}$ produces a first rearrangement, which primes $\mathrm{VP}^{\star}{ }^{*}$ for membrane attack (Fig. 4b). Dimeric interactions in the external portion of two primed VP4 subunits rigidify the spikes, elevating and presenting the $\mathrm{VP}{ }^{\star}$ core for cell-surface ligand binding. The third VP4 molecule remains flexible. The heads of the spike shield hydrophobic prominences on the body. We hypothesize that a subsequent, as yet unknown, entryassociated event triggers a transition to the trimeric conformation seen in the crystal structure (Fig. 4c). In this second transition, disruption of the dimer contacts releases $\mathrm{VP}^{\star}$ from $\mathrm{VP} 5^{\star}$ and unmasks the hydrophobic apex of the VP5 ${ }^{\star}$ antigen domain, which may insert into a host-cell membrane. As the trimeric coiled-coil zips up, the antigen domain folds back against it. The resulting translocation of the potential membrane-interaction loop towards the foot could disrupt a cellular membrane. This disruption may in turn create a breach that allows a subviral particle to enter the cytoplasm, or it could lower the local calcium concentration, leading to virion uncoating and subsequent entry events. The fold-back transition could also alter interactions with receptors or directly trigger virion uncoating.

The essential properties of VP4-its capacity to adopt an inactive, precursor conformation; a primed, receptor-binding conformation; and a final, folded-back conformation-recall those of enveloped virus fusion proteins $s^{3-6}$. Understanding these transformations will allow us to analyse neutralization mechanisms, design subunit immunogens, and elucidate molecular details of membrane perforation.

\section{Methods}

\section{Expression and purification of RRV VP5CT}

VP5CT was produced by serial chymotrypsin and trypsin digestion of purified RRV VP4 from recombinant baculovirus-infected insect cells, as described previously ${ }^{13}$.

Selenomethionine-substituted protein was produced by metabolic incorporation in insect cells $^{20}$.

\section{Crystallization}

VP5CT crystallized at $23^{\circ} \mathrm{C}$ in hanging drops containing equal volumes of well solutions and a protein solution containing $9 \mathrm{mg} \mathrm{ml}^{-1}$ VP5CT, $20 \mathrm{mM}$ Tris, $\mathrm{pH} 8.0,1 \mathrm{mM}$ EDTA, $0.02 \%$ sodium azide and $0.1 \mathrm{mM}$ benzamidine. Selenomethionine-substituted VP5CT crystals that formed after 4 days with a well solution of $6 \%$ ethanol, $50 \mathrm{mM}$ Tris $\mathrm{pH} 7.0$ and $1 \mathrm{mM}$ dithiothreitol were harvested after one month and flash-frozen with liquid nitrogen in a freezing buffer of $11 \%$ ethanol, $50 \mathrm{mM}$ Tris, $\mathrm{pH}$ 7.0, 17\% 2-methyl-2,4-pentanediol (MPD) and $5 \mathrm{mM}$ dithiothreitol. Unsubstituted VP5CT crystals that formed after 3 weeks with a well solution of $500 \mathrm{mM}$ ammonium sulphate, 5.5-6\% MPD and $50 \mathrm{mM}$ PIPES pH 6.5 were harvested after 1-7 months and flash-frozen in $600 \mathrm{mM}$ ammonium sulphate, $22.5 \% \mathrm{MPD}$ and $20-50 \mathrm{mM}$ Tris, $\mathrm{pH}$ 8.0.

\section{Structure determination}

Diffraction data were scaled in space group $P 4(2) 22$, with six monomers per asymmetric unit. Starting phases were determined by multiple wavelength anomalous dispersion, based on diffraction from crystals of selenomethionine-substituted VP5CT. Thirty seleniums per asymmetric unit were located by direct methods, using $\mathrm{SnB}^{21}$. Landmarks provided by seleniums and bulky side chains unambiguously aligned the primary amino acid sequence to the electron density. Refinement took advantage of six-fold noncrystallographic symmetry. The final model contains no Ramachandran violations, and $76.6 \%$ of dihedral angles are in most-favoured regions. Pseudocentring and perfect hemihedral twinning in VP5CT crystals has been described previously ${ }^{22}$. Details of the structure determination are provided in Supplementary Methods, Supplementary Table 4, and Supplementary Fig. 2.

\section{Electron cryomicroscopy}

Electron micrographs of rotavirus particles (strain SA11-4F) embedded in a thin layer of vitreous ice were recorded under low electron dose conditions $\left(\sim 10 e^{-} \AA^{-2}\right)$ at $\times 50,000$ magnification on a JEOL 4000 electron microscope operated at $400 \mathrm{kV}$. Electron

micrographs were digitized with a scanning interval corresponding to $2.8 \AA$ in the object. A three-dimensional reconstruction was computed using cylindrical expansion methods ${ }^{23,24}$ to $12.4 \AA$ resolution by combining 908 particles from 15 micrographs with appropriate corrections for contrast transfer function. Defocus values, determined using EMAN software ${ }^{25}$, ranged from 0.8 to $3-\mu \mathrm{m}$ underfocus. As is conventional ${ }^{23}$, to compute initial maps, electron microscopy data were first analysed in reciprocal space, using only 5-2-2 symmetry. To compute the final map, full icosahedral symmetry was imposed by threefold real-space averaging after confirming the presence of three-fold symmetry in initial maps. The protruding portion of the VP4 spike was computationally extracted from the final density map. Crystal structures were fit to the electron cryomicroscopy map by a combination of automated searching using Situs ${ }^{26}$ and manual fitting.

\section{Illustration}

Figures were made using $\mathrm{O}^{27}$, Molscript ${ }^{28}$, Grasp ${ }^{29}$, Photoshop (Adobe Systems) and Illustrator (Adobe Systems).

Received 10 April; accepted 14 July 2004; doi:10.1038/nature02836.

1. Parashar, U. D., Hummelman, E. G., Bresee, J. S., Miller, M. A. \& Glass, R. I. Global illness and deaths caused by rotavirus disease in children. Emerg. Infect. Dis. 9, 565-572 (2003).

2. Crawford, S. E. et al. Trypsin cleavage stabilizes the rotavirus VP4 spike. J. Virol. 75, 6052-6061 (2001).

3. Ruigrok, R. W. et al. Studies on the structure of the influenza virus haemagglutinin at the $\mathrm{pH}$ of membrane fusion. J. Gen. Virol. 69, 2785-2795 (1988). 
4. Weissenhorn, W. et al. The ectodomain of HIV-1 env subunit gp41 forms a soluble, alpha-helical, rod-like oligomer in the absence of gp 120 and the $\mathrm{N}$-terminal fusion peptide. EMBO J. 15, 1507-1514 (1996).

Modis, Y., Ogata, S., Clements, D. \& Harrison, S. C. Structure of the dengue virus envelope protein after membrane fusion. Nature 427, 313-319 (2004)

6. Gibbons, D. L. et al. Conformational change and protein-protein interactions of the fusion protein of Semliki Forest virus. Nature 427, 320-325 (2004).

7. Dormitzer, P. R., Sun, Z.-Y. J., Wagner, G. \& Harrison, S. C. The rhesus rotavirus VP4 sialic acid binding domain has a galectin fold with a novel carbohydrate binding site. EMBO J. 21, 885-897 (2002).

8. Mackow, E. R. et al. The rhesus rotavirus gene encoding protein VP3: location of amino acids involved in homologous and heterologous rotavirus neutralization and identification of a putative fusion region. Proc. Natl Acad. Sci. USA 85, 645-649 (1988).

9. Dowling, W., Denisova, E., LaMonica, R. \& Mackow, E. R. Selective membrane permeabilization by the rotavirus VP5* protein is abrogated by mutations in an internal hydrophobic domain. J. Virol. 74, 6368-6376 (2000).

10. Graham, K. L. et al. Integrin-using rotaviruses bind $\alpha 2 \beta 1$ integrin $\alpha 2$ I domain via VP4 DGE sequence and recognize $\alpha \mathrm{X} \beta 2$ and $\alpha \mathrm{V} \beta 3$ by using VP7 during cell entry. J. Virol. 77, 9969-9978 (2003).

11. Ruggeri, F. M. \& Greenberg, H. B. Antibodies to the trypsin cleavage peptide VP8 neutralize rotavirus by inhibiting binding of virions to target cells in culture. J. Virol. 65, 2211-2219 (1991).

12. Offit, P. A., Shaw, R. D. \& Greenberg, H. B. Passive protection against rotavirus-induced diarrhea by monoclonal antibodies to surface proteins vp3 and vp7. J. Virol. 58, 700-703 (1986).

13. Dormitzer, P. R., Greenberg, H. B. \& Harrison, S. C. Proteolysis of monomeric recombinant rotaviru VP4 yields an oligomeric VP5* core. J. Virol. 75, 7339-7350 (2001)

14. Arias, C. F., Romero, P., Alvarez, V. \& Lopez, S. Trypsin activation pathway of rotavirus infectivity. J. Virol. 70, 5832-5839 (1996).

15. Gilbert, J. M. \& Greenberg, H. B. Cleavage of rhesus rotavirus VP4 after arginine 247 is essential for rotavirus-like particle-induced fusion from without. J. Virol. 72, 5323-5327 (1998)

16. Tihova, M., Dryden, K. A., Bellamy, A. R., Greenberg, H. B. \& Yeager, M. Localization of membrane permeabilization and receptor binding sites on the VP4 hemagglutinin of rotavirus: implications for cell entry. J. Mol. Biol. 314, 985-992 (2001).

17. Taniguchi, K. et al. Identification of cross-reactive and serotype 2-specific neutralization epitopes on VP3 of human rotavirus. J. Virol. 62, 2421-2426 (1988).

18. Shaw, A. L. et al. Three-dimensional visualization of the rotavirus hemagglutinin structure. Cell 74, 693-701 (1993).

19. Yeager, M., Berriman, J. A., Baker, T. S. \& Bellamy, A. R. Three-dimensional structure of the rotaviru haemagglutinin VP4 by cryo-electron microscopy and difference map analysis. EMBO J. 13, 1011-1018 (1994).

20. Liemann, S., Chandran, K., Baker, T. S., Nibert, M. L. \& Harrison, S. C. Structure of the reovirus membrane-penetration protein, $\mu 1$, in a complex with its protector protein, $\sigma 3$. Cell 108, 283-295 (2002).

21. Weeks, C. M. \& Miller, R. The design and implementation of SnB v2.0. J. Appl. Crystallogr. 32, 120-124 (1999).

22. Padilla, J. E. \& Yeates, T. O. A statistic for local intensity differences: robustness to anisotropy and pseudo-centering and utility for detecting twinning. Acta Crystallogr. D 59, 1124-1130 (2003).

23. Crowther, R. A. Procedures for three-dimensional reconstruction of spherical viruses by Fourier synthesis from electron micrographs. Phil. Trans. R. Soc. Lond. B 261, 221-230 (1971).

24. Lawton, J. A. \& Prasad, B. V. V. Automated software package for icosahedral virus reconstruction. J. Struct. Biol. 116, 209-215 (1996).

25. Ludtke, S. J., Baldwin, P. R. \& Chiu, W. EMAN: semiautomated software for high-resolution singleparticle reconstructions. J. Struct. Biol. 128, 82-97 (1999).

26. Wriggers, W., Milligan, R. A. \& McCammon, J. A. Situs: A package for docking crystal structures into low-resolution maps from electron microscopy. J. Struct. Biol. 125, 185-195 (1999).

27. Jones, T. A., Zou, J. Y., Cowan, S. W. \& Kjeldgaard, M. Improved methods for binding protein model in electron density maps and the location of errors in these models. Acta Crystallogr. A 47, 110-119 (1991).

28. Kraulis, J. MOLSCRIPT: A program to produce both detailed and schematic plots of protein structures. J. Appl. Crystallogr. 24, 946-950 (1991)

29. Nicholls, A., Sharp, K. A. \& Honig, B. Protein folding and association: insights from interfacial and thermodynamic properties of hydrocarbons. Prot. Struct. Funct. Genet. 11, 281-296 (1991).

30. Yeager, M., Dryden, K. A., Olson, N. H., Greenberg, H. B. \& Baker, T. S. Three-dimensional structure of rhesus rotavirus by cryoelectron microscopy and image reconstruction. J. Cell Biol. 110, 2133-2144 (1990).

Supplementary Information accompanies the paper on www.nature.com/nature.

Acknowledgements We thank M. Babyonyshev for technical assistance; T. Yeates for help in analysing the crystal twinning disorder; $\mathrm{H}$. Greenberg for cloned genes and recombinant baculoviruses; E. Vogan for help with data collection and analysis; and the staff of Advanced Photon Source beamline ID-19 (Argonne National Laboratory) and Cornell High Energy Synchrotron Source beamlines Fl and Al. We acknowledge the use of electron cryomicroscopy facilities at the National Center for Macromolecular Imaging funded by NIH at Baylor College of Medicine. This work was supported by an NIH grant and an Ellison Medical Foundation New Investigator in Global Infectious Diseases award to P.R.D., by an NIH grant to B.V.V.P., and by an NIH grant to S.C.H., who is a Howard Hughes Medical Institute Investigator

Competing interests statement The authors declare that they have no competing financial interests.

Correspondence and requests for materials should be addressed to P.R.D.

(dormitze@crystal.harvard.edu).Coordinates and observed structure factors have been deposited in the Protein Data Bank under accession code 1SLQ.

\section{The native architecture of a photosynthetic membrane}

\author{
Svetlana Bahatyrova ${ }^{1 \star}$, Raoul N. Frese ${ }^{1,2 \star}$, C. Alistair Siebert ${ }^{3}$, \\ John D. Olsen ${ }^{3}$, Kees O. van der Werf ${ }^{1}$, Rienk van Grondelle ${ }^{2}$, \\ Robert A. Niederman ${ }^{4}$, Per A. Bullough ${ }^{3}$, Cees Otto $^{1}$ \& C. Neil Hunter ${ }^{3}$
}

${ }^{1}$ Biophysical Techniques Group, Department of Science \& Technology, BMTI, $\mathrm{MESA}^{+}$, University of Twente, P.O. Box 217, 7500 AE Enschede, The Netherlands ${ }^{2}$ Biophysics, Faculty of Sciences, Vrije Universiteit Amsterdam, de Boelelaan 1081, $1081 \mathrm{HV}$, The Netherlands

${ }^{3}$ Department of Molecular Biology and Biotechnology, University of Sheffield, Sheffield S10 2TN, UK

${ }^{4}$ Rutgers University, Department of Molecular Biology and Biochemistry,

Piscataway, New Jersey 08854, USA

* These authors contributed equally to this work

In photosynthesis, the harvesting of solar energy and its subsequent conversion into a stable charge separation are dependent upon an interconnected macromolecular network of membraneassociated chlorophyll-protein complexes. Although the detailed structure of each complex has been determined ${ }^{1-4}$, the size and organization of this network are unknown. Here we show the use of atomic force microscopy to directly reveal a native bacterial photosynthetic membrane. This first view of any multi-component membrane shows the relative positions and associations of the photosynthetic complexes and reveals crucial new features of the organization of the network: we found that the membrane is divided into specialized domains each with a different network organization and in which one type of complex predominates. Two types of organization were found for the peripheral light-harvesting LH2 complex. In the first, groups of 10-20 molecules of LH2 form light-capture domains that interconnect linear arrays of dimers of core reaction centre (RC)-light-harvesting 1 (RC-LH1-PufX) complexes; in the second they were found outside these arrays in larger clusters. The LH1 complex is ideally positioned to function as an energy collection hub, temporarily storing it before transfer to the RC where photochemistry occurs: the elegant economy of the photosynthetic membrane is demonstrated by the close packing of these linear arrays, which are often only separated by narrow 'energy conduits' of LH2 just two or three complexes wide.

Photosynthetic purple bacteria can contain two types of complex, RC-LH1 and LH2, with both light-harvesting complexes comprising roughly circularly arranged $\alpha$-helices with bound carotenoid and bacteriochlorophyll (Bchl) pigments ${ }^{3-6}$. To investigate the functionally crucial organization of these complexes, native photosynthetic membranes from the wild-type purple bacterium Rhodobacter sphaeroides were imaged by atomic force microscopy (AFM), a technique that allows the topography of biological samples to be acquired in buffer solution at room temperature and under normal pressure. Figure 1a shows a cluster of several membrane patches, each of a size approximating to the surface area of an intracytoplasmic membrane vesicle (chromatophore). The bright areas represent photosynthetic complexes; even at this low magnification this remarkable view of native photosynthetic membranes shows that they are composed, at least in part, of linear arrays of dimeric complexes. This arrangement was mirrored in all the membrane patches we examined, and a gallery of additional images is shown in Supplementary Fig. S1.

Figure 1b clearly shows how the light-harvesting and photochemical functions of a membrane are apportioned, and reveals the arrangement of photosynthetic complexes. Two types can be seen: large circular complexes with a bright and therefore 